\title{
Anxiety disorders and inflammation in a large adult cohort
}

\author{
N Vogelzangs ${ }^{1}$, ATF Beekman ${ }^{1}, \mathrm{P}$ de Jonge ${ }^{2}$ and BWJH Penninx $x^{1,2,3}$
}

\begin{abstract}
Although anxiety disorders, like depression, are increasingly being associated with metabolic and cardiovascular burden, in contrast with depression, the role of inflammation in anxiety has sparsely been examined. This large cohort study examines the association between anxiety disorders and anxiety characteristics with several inflammatory markers. For this purpose, persons (18-65 years) with a current $(N=1273)$ or remitted $(N=459)$ anxiety disorder (generalized anxiety disorder, social phobia, panic disorder, agoraphobia) according to Diagnostic and Statistical Manual of Mental Disorders, Fourth Edition criteria and healthy controls $(N=556)$ were selected from the Netherlands Study of Depression and Anxiety. In addition, severity, duration, age of onset, anxiety subtype and co-morbid depression were assessed. Inflammatory markers included C-reactive protein (CRP), interleukin (IL)- 6 and tumor-necrosis factor (TNF)- $\alpha$. Results show that after adjustment for sociodemographics, lifestyle and disease, elevated levels of CRP were found in men, but not in women, with a current anxiety disorder compared with controls $\left(1.18\right.$ (s.e. $=1.05$ ) versus 0.98 (s.e. $=1.07$ ) $\mathrm{mg} \mathrm{I}^{-1}, P=0.04$, Cohen's $d=0.18$ ). No associations were found with IL- 6 or TNF- $\alpha$. Among persons with a current anxiety disorder, those with social phobia, in particular women, had lower levels of CRP and IL-6, whereas highest CRP levels were found in those with an older age of anxiety disorder onset. Especially in persons with an age of onset after 50 years, CRP levels were increased compared with controls $(1.95$ (s.e. $=1.18)$ versus $1.27($ s.e. $=1.05) \mathrm{mg} \mathrm{I}^{-1}$, $P=0.01$, Cohen's $d=0.37$ ). In conclusion, elevated inflammation is present in men with current anxiety disorders. Immune dysregulation is especially found in persons with a late-onset anxiety disorder, suggesting the existence of a specific late-onset anxiety subtype with a distinct etiology, which could possibly benefit from alternative treatments.
\end{abstract}

Translational Psychiatry (2013) 3, e249; doi:10.1038/tp.2013.27; published online 23 April 2013

\section{Introduction}

Anxiety disorders are among the most prevalent and disabling mental disorders. ${ }^{1,2}$ Increasing evidence links anxiety to cardiovascular risk factors and diseases such as atherosclerosis, ${ }^{3}$ metabolic syndrome, ${ }^{4}$ and coronary heart disease. $^{5,6}$ As low-grade systemic inflammation is clearly involved in the etiology of these somatic conditions, ${ }^{7-9}$ it has been hypothesized that inflammation has a role in anxiety disorders and may form the link between anxiety disorders and cardiovascular burden. ${ }^{10}$ Anxiety disorders are also highly co-morbid with depression, ${ }^{11}$ which has recurrently been associated with immune dysregulation. ${ }^{12,13}$ However, unlike depression, very few studies have investigated the relationship between anxiety disorders and inflammation. Two recent studies have correlated anxiety symptoms with increased cytokine levels, in particular C-reactive protein (CRP). ${ }^{14,15}$ With regard to anxiety disorders, research has mainly focused on posttraumatic stress disorder, in which high levels of inflammatory markers have been found. ${ }^{16,17}$ Sparse evidence from relatively small clinical studies $(n \approx 100)$ suggests increased inflammatory activation in patients with panic disorder ${ }^{18}$ and generalized anxiety disorder, ${ }^{19}$ which seems to be independent of co-morbid depression.
As there is yet limited research on immune dysregulation and anxiety, one can only speculate on the mechanisms linking these two conditions. Experimentally induced stress has been shown to produce an inflammatory reaction, ${ }^{20}$ which has led researchers to suggest that it is in particular the experience of acute stress, such as present in panic disorders, causing the high levels of inflammation in anxiety. ${ }^{18}$ On the other hand, chronic stress may initiate changes in the hypothalamic-pituitary-adrenal (HPA) axis and the immune system, which in turn can trigger depression as well as anxiety. ${ }^{21}$ These pathways are not independent as the HPAaxis and the immune system are closely linked. Although the HPA axis in normal situations should temper inflammatory reactions, prolonged hyperactivity of the HPA axis could result in blunted anti-inflammatory responses to glucocorticoids resulting in increased inflammation. ${ }^{22,23}$ Likewise, it can be hypothesized that immune changes associated with chronic disease and aging, ${ }^{24}$ could induce similar anxiety-enhancing effects. Although several mechanisms might explain an association between inflammation and anxiety disorders, it can be expected that immune dysregulation is not a general phenomenon in anxiety disorders, but might be restricted to specific subgroups. Whether this anxiety subgroup is defined by the type of

${ }^{1}$ Department of Psychiatry, EMGO Institute for Health and Care Research, and Neuroscience Campus Amsterdam, VU University Medical Center, Amsterdam, The Netherlands; ' ${ }^{2}$ Department of Psychiatry, University Medical Center Groningen, Groningen, The Netherlands and ${ }^{3}$ Department of Psychiatry, Leiden University Medical Center, Leiden, The Netherlands

Correspondence: Dr N Vogelzangs, Department of Psychiatry, EMGO Institute for Health and Care Research, VU University Medical Center, AJ Ernststraat 1187, 1081 HL Amsterdam, The Netherlands.

E-mail: n.vogelzangs@vumc.nl or n.vogelzangs@ggzingeest.nl

Keywords: anxiety disorder; anxiety characteristics; cohort study; inflammation

Received 4 October 2012; revised 21 February 2013; accepted 15 March 2013 
disorder, the severity or duration of the disorder, the co-morbidity with depression, or its age of onset, is yet to be examined.

The present study investigates the association between several common anxiety disorders (generalized anxiety disorder, social phobia, panic disorder, agoraphobia) and heightened inflammation (CRP, interleukin (IL)-6, tumor necrosis factor (TNF)- $\alpha$ ) in a large sample of persons with current and remitted anxiety disorders and healthy controls. In addition, it will be examined whether specific anxiety characteristics (severity, duration, age of onset, subtype, depression co-morbidity) further discriminate those anxiety patients with elevated inflammation.

\section{Subjects and methods}

Sample. The Netherlands Study of Depression and Anxiety (NESDA) includes 2981 persons with and without depressive and anxiety disorders, aged 18-65 years at the baseline assessment in 2004-2007. Participants were recruited from the community (19\%), general practice (54\%) and secondary mental health care $(27 \%)$ in order to reflect the broad range and developmental trajectory of psychopathology. Persons with insufficient command of the Dutch language or a primary clinical diagnosis of bipolar disorder, obsessive compulsive disorder, severe substance use disorder, psychotic disorder or organic psychiatric disorder, as reported by themselves or their mental health practitioner, were excluded. A detailed description of the NESDA study design and sampling procedures can be found elsewhere. ${ }^{25}$ The research protocol was approved by the ethics committee of participating universities and after complete description of the study all respondents provided written informed consent.

During the baseline interview, the presence of anxiety disorder (generalized anxiety disorder, social phobia, panic disorder, agoraphobia) and depressive disorder (major depressive disorder, dysthymia) was established using the Composite Interview Diagnostic Instrument (CIDI) according to Diagnostic and Statistical Manual of Mental Disorders, Fourth Edition criteria. ${ }^{26}$ The CIDI is a highly reliable and valid instrument for assessing depressive and anxiety disorders ${ }^{27}$ and was administered by specially trained research staff. In addition, the severity of anxiety was measured in all participants using the 21-item self-report Beck Anxiety Inventory. ${ }^{28}$ For the present analyses, we selected persons with a current (that is, past 6 months) or remitted (lifetime, but not current) anxiety disorder and healthy controls. Healthy controls had no lifetime anxiety or depressive disorder and a Beck Anxiety Inventory score below 10, as a score of 10 or above indicates mild anxiety. ${ }^{29}$ Persons with anxiety disorders were allowed to have a co-morbid depression. Of these 2342 persons, 54 were excluded due to missing information on inflammatory markers, leaving a sample of 2288 persons for the present study. Persons with missing data on inflammation were less often female (55.6 versus $66.9 \%, P=0.08$ ), but did not differ from included persons in terms of age, years of education and the presence of anxiety disorder.

Anxiety characteristics. Next to subtype of CIDI anxiety disorder diagnosis (generalized anxiety disorder, social phobia, panic disorder, agoraphobia), anxiety characteristics included anxiety symptoms severity as measured by the Beck Anxiety Inventory, and anxiety symptoms duration, using the Life Chart Interview (LCI). ${ }^{30}$ The LCl uses a calendar method to determine life events during the past 4 years to refresh memory after which the presence of anxiety and avoidance symptoms during that period is assessed. From this, the per cent of time patients reported anxiety symptoms was computed. The $\mathrm{LCl}$ has been used by other large cohort studies ${ }^{31}$ and event history calendars such as the $\mathrm{LCl}$ have been suggested a natural method of choice for retrospective data collection. ${ }^{32}$ To be able to test whether inflammation was in particular associated with anxiety disorders with a later onset, as we had found for depression, ${ }^{33}$ age of anxiety onset was derived from the CIDI interview. Last, the presence of a current co-morbid depressive disorder (major depressive disorder, dysthymia) was taken from the CIDI to check whether a possible inflammation-anxiety association was independent of comorbid depression.

Inflammatory markers. Markers of inflammation were assessed at the baseline NESDA measurement and included CRP, IL- 6 and TNF- $\alpha$. Fasting blood samples of NESDA participants were obtained in the morning between 0800 and 0900 hours and kept frozen at $-80^{\circ} \mathrm{C}$. CRP and IL- 6 were assayed at the Clinical Chemistry Department of the VU University Medical Center. High-sensitivity plasma levels of CRP were measured in duplicate by an in-house ELISA based on purified protein and polyclonal anti-CRP antibodies (Dako, Glostrup, Denmark). Intra- and inter-assay coefficients of variation were $5 \%$ and $10 \%$, respectively. Plasma IL-6 levels were measured in duplicate by a high sensitivity ELISA (PeliKine Compact ${ }^{\mathrm{TM}}$ ELISA, Sanquin, Amsterdam, The Netherlands). Intra- and inter-assay coefficients of variation were $8 \%$ and $12 \%$, respectively. Plasma TNF- $\alpha$ levels were assayed in duplicate at Good Biomarker Science, Leiden, The Netherlands, using a high-sensitivity solid phase ELISA (Quantikine ${ }^{\circledR}$ HS Human TNF- $\alpha$ Immunoassay, R\&D systems, Minneapolis, MN, USA). Intra- and inter-assay coefficients of variation were $10 \%$ and $15 \%$, respectively.

Covariates. Sociodemographic characteristics included sex, age and years of education. As lifestyle characteristics can be associated with both anxiety and inflammation, smoking status (never, former, current), alcohol intake $(<1$, 1-14 (women)/1-21 (men), > 14 (women)/> 21 (men) drinks per week), physical activity (measured with the International Physical Activity Questionnaire ${ }^{34}$ in MET-minutes (ratio of energy expenditure during activity compared with rest times the number of minutes performing the activity) per week) and body mass index (weight in kilograms divided by height in meters squared) were assessed. In addition, several disease-related covariates were taken into account including the presence of cardiovascular disease (assessed by selfreport supported by appropriate medication use (see Vogelzangs et al. ${ }^{6}$ for detailed description)), the presence of diabetes (fasting plasma glucose level $\geqslant 7.0 \mathrm{mmoll}^{-1}$ or use of anti-diabetic medication (ATC code A10)) and the number of other self-reported chronic diseases for which 
persons received treatment (including lung disease, osteoarthritis or rheumatic disease, cancer, ulcer, intestinal problem, liver disease, epilepsy and thyroid gland disease). Medication use was assessed based on drug container inspection of all drugs used in the past month and classified according to the World Health Organization Anatomical Therapeutic Chemical classification. ${ }^{35}$ Statin use (C10AA, C10B) and use of systemic anti-inflammatory medication (M01A, M01B, A07EB, A07EC) were assessed. Antidepressant medication included regular use ( $>50 \%$ of the time) of selective serotonin reuptake inhibitors (SSRI; N06AB), serotoninnorepinephrine reuptake inhibitors (SNRI; N06AX16, N06AX21), tricyclic antidepressants (TCA; N06AA) and tetracyclic antidepressants (TeCA; N06AX03, N06AX05, N06AX11).

Statistical analyses. Baseline characteristics were compared between men and women using $\chi^{2}$ test for dichotomous and categorical variables, independent samples $t$-test for continuous variables, and Mann-Whitney $U$-test for inflammatory markers. For subsequent analyses, CRP, IL-6 and TNF- $\alpha$ were In-transformed to normalize distributions, but back-transformed values are presented to enhance interpretation. Associations between anxiety disorders and inflammatory markers were examined using analyses of (co)variance, and (adjusted) means across anxiety groups (no, remitted, current) are presented. To take the effects of potential confounding factors into account, three different models were tested: unadjusted, adjusted for sociodemographics (sex, age, education) and additionally adjusted for lifestyle and disease (smoking status, alcohol intake, physical activity, body mass index, cardiovascular disease, diabetes, number of other chronic diseases, statins, anti-inflammatory medication). As depression has been reported to differentially affect inflammation in men and women, ${ }^{33}$ a sex-interaction for anxiety disorders is plausible. Therefore, we tested sex-interactions by including a sex $\times$ anxiety disorder status interaction term. When present, analyses were repeated sex stratified.

To test whether specific anxiety characteristics were related to elevated inflammation levels, we performed linear regression analyses with inflammatory markers as the outcome for each anxiety characteristic (severity, duration, age of onset, subtype, depression co-morbidity) within the sample of persons with a current anxiety disorder.

\section{Results}

Mean age of the study sample was 41.8 (s.d. = 13.1) years and $66.9 \%$ were women. Baseline characteristics of the total sample and for men and women separately are shown in Table 1. Women were younger, more often non-drinkers, had a lower body mass index, less often cardiovascular disease or diabetes and less often used statins than men. In addition, women had higher levels of CRP than men. All covariates were associated with at least one of the inflammation markers, which has been presented elsewhere. ${ }^{33}$ Pearson's correlations between inflammatory markers were modest (CRP-IL-6: $r=0.31$; CRP-TNF$\alpha: r=0.13$; IL-6-TNF- $\alpha: r=0.12$; all $P<0.001)$.

Table 2 shows (adjusted) mean inflammation levels across anxiety groups (controls, remitted, current) based on analyses of (co)variance. In the total sample, higher CRP levels were found in persons with a current anxiety disorder compared with controls in unadjusted analyses $(1.36$ (s.e. $=1.04$ ) versus 1.11 (s.e. $=1.05) \mathrm{mgl}^{-1}, P=0.001$ ), but after adjustment, there were no associations between anxiety disorders and any of the inflammation markers. However, a significant sex $\times$ anxiety disorder interaction was found for CRP (remitted: $P=0.57$; current: $P=0.002$ ). Stratified analyses for CRP showed that even after full adjustment for lifestyle and disease, men with current anxiety disorders had higher levels of CRP compared with controls (1.18 (s.e. $=1.05)$ versus 0.98 (s.e. $=1.07$ ) $\mathrm{mg} \mathrm{I}^{-1}, P=0.04$, Cohen's $\mathrm{d}=0.18$ ). In women, anxiety disorders were not significantly associated with CRP. No sex interactions were found for IL-6 (remitted: $P=0.47$; current: $P=0.40$ ) or TNF- $\alpha$ (remitted: $P=0.92$; current: $P=0.87$ ). As we have previously reported associations between inflammatory levels and antidepressant use within currently depressed persons, ${ }^{33}$ we checked the influence of antidepressant use on our current results. Higher levels of CRP were found in TCA/TeCA users within our present sample of persons with current anxiety disorders $(N=1273$; $P=0.001$ ). To examine whether the finding of elevated CRP in currently anxious men was independent of TCA/TeCA use, we excluded all men using TCA/TeCA $(N=36)$. Results remained similar, although no longer significant (men with current anxiety disorders versus controls: 1.13 (s.e. $=1.05$ ) versus 0.97 (s.e. $=1.07$ ) $\mathrm{mg} \mathrm{l}^{-1}, P=0.08$, Cohen's $d=0.15$ ). In addition, to reduce the possible confounding effects of acute illness on inflammatory levels at the time of blood draw, all persons who reported having had a cold or fever in the week before blood draw were excluded $(N=645)$, but findings remained alike (men with current anxiety disorders versus controls: 1.09 (s.e. $=1.06$ ) versus 0.91 (s.e. $=1.07$ ) $\mathrm{mgl}^{-1}$, $P=0.06$, Cohen's $d=0.19$ ).

To investigate whether specific anxiety characteristics (severity, duration, age of onset, subtype, depression comorbidity) were associated with inflammation, linear regression analyses were performed within the subgroup of persons with current anxiety disorders $(N=1273$; Table 3$)$. Anxiety severity and duration did not correlate with inflammation. Later age of anxiety disorder onset was associated with elevated CRP levels $(\beta=0.053, P=0.05)$, even after additional adjustment for TCA/TeCA use $(\beta=0.053, P=0.05)$. Persons with social phobia had lower levels of CRP $(\beta=-0.053, P=0.04)$ and IL-6 $(\beta=-0.052, P=0.05)$ compared with persons with other types of anxiety disorders. The association between social phobia and IL-6 appeared to be specific for women $(\beta=-0.089, P=0.007)$, but not men ( $\beta=0.025, P=0.61 ; P$ sex-interaction $=0.05)$. Co-morbid depressive disorder did not further differentiate anxious persons with elevated inflammation.

To further illustrate the findings with regard to age of onset, we constructed five age of anxiety disorder onset groups $(<20,20-30,30-40,40-50, \geqslant 50)$. Figure 1 presents adjusted means of back-transformed CRP levels across controls and age of onset groups based on analysis of covariance. CRP levels were only increased in persons with an age of onset after 50 years (1.95 (s.e. $=1.18$ ) versus 1.27 (s.e. $=1.05$ ) $\mathrm{mg} \mathrm{l}^{-1}$ in controls, $P=0.01$, Cohen's $d=0.37$ ). For comparison, adjusted mean CRP levels for persons with 
Table 1 Baseline characteristics

\begin{tabular}{|c|c|c|c|c|}
\hline & Total sample $(\mathrm{N}=2288)$ & $\operatorname{Men}(\mathrm{N}=757)$ & Women $(\mathrm{N}=1531)$ & P-values ${ }^{\mathrm{a}}$ \\
\hline \multicolumn{5}{|l|}{ Sociodemographics } \\
\hline Age (years), mean (s.d.) & $41.8(13.1)$ & $43.2(13.1)$ & $41.1(13.0)$ & 0.001 \\
\hline Education (years), mean (s.d.) & $12.1(3.3)^{\prime}$ & $12.2(3.3)^{\prime}$ & $12.1(3.2)$ & 0.67 \\
\hline \multicolumn{5}{|l|}{ Lifestyle and disease } \\
\hline Smoking status & & & & 0.14 \\
\hline Never, \% & 28.3 & 25.6 & 29.6 & \\
\hline Former, \% & 33.5 & 34.3 & 33.1 & \\
\hline Current, \% & 38.2 & 40.0 & 37.4 & \\
\hline Alcohol intake & & & & $<0.001$ \\
\hline$<1$ Drink a week, \% & 32.5 & 21.1 & 38.1 & \\
\hline $1-14$ (women)/1-21 (men) drinks a week, \% & 56.3 & 67.5 & 50.8 & \\
\hline$>14($ women) $/>21$ (men) drinks a week, \% & 11.2 & 11.4 & 11.2 & \\
\hline Physical activity (MET-minutes/week), mean (s.d.) & 3644 (2999) & $3711(3304)$ & $3611(28361)$ & 0.48 \\
\hline Body mass index, mean (s.d.) & $25.6(5.1)$ & $26.1(4.5)$ & $25.3(5.3)$ & $<0.001$ \\
\hline Cardiovascular disease, $\%$ & 6.2 & 10.0 & 4.2 & $<0.001$ \\
\hline Diabetes, \% & 4.9 & 7.4 & 3.6 & $<0.001$ \\
\hline Number of other chronic diseases, mean (s.d.) & $0.4(0.7)$ & $0.4(0.7)$ & $0.4(0.7)$ & 0.14 \\
\hline Statin, \% & 6.4 & 9.5 & 4.9 & $<0.001$ \\
\hline Anti-inflammatory medication, \% & 4.1 & 3.0 & 4.6 & 0.07 \\
\hline \multicolumn{5}{|l|}{ Anxiety characteristics } \\
\hline Anxiety disorder & & & & 0.001 \\
\hline Control, \% & 24.3 & 28.9 & 22.0 & \\
\hline Remitted anxiety disorder, \% & 20.1 & 17.3 & 21.4 & \\
\hline Current anxiety disorder, \% & 55.6 & 53.8 & 56.6 & \\
\hline \multicolumn{5}{|l|}{ Within persons with a current anxiety disorder $(\mathrm{N}=1273)$} \\
\hline Severity (BAI score), mean (s.d.) & $18.4(10.9)$ & $17.6(10.7)$ & $18.8(10.9)$ & 0.06 \\
\hline Duration (\% of time), mean (s.d.) & $56.6(36.3)$ & $58.7(36.5)$ & $55.6(36.3)$ & 0.17 \\
\hline Age of anxiety onset (years), mean (s.d.) & $21.2(12.8)$ & $23.2(13.9)$ & $20.2(12.1)$ & $<0.001$ \\
\hline Generalized anxiety disorder, \% & 35.7 & 38.6 & 34.3 & 0.14 \\
\hline Social phobia, \% & 51.1 & 51.1 & 51.0 & 0.98 \\
\hline Panic disorder and/or agoraphobia, \% & 65.4 & 61.7 & 67.2 & 0.05 \\
\hline Co-morbid depressive disorder, \% & 58.4 & 57.2 & 59.0 & 0.55 \\
\hline \multicolumn{5}{|l|}{ Inflammatory markers } \\
\hline C-reactive protein $\left(\mathrm{mgl}^{-1}\right)$, median (IQR) & $1.20(0.54-3.00)$ & $1.01(0.48-2.40)$ & $1.33(0.57-3.30)$ & $<0.001$ \\
\hline Interleukin-6 (pg ml- $\left.{ }^{-1}\right)$, median (IQR) & $0.75(0.49-1.25)$ & $0.77(0.51-1.31)$ & $0.75(0.49-1.24)$ & 0.25 \\
\hline Tumor necrosis factor- $\alpha\left(\mathrm{pg} \mathrm{ml}^{-1}\right)$, median (IQR) & $0.80(0.60-1.10)$ & $0.80(0.60-1.10)$ & $0.70(0.60-1.10)$ & 0.13 \\
\hline
\end{tabular}

Abbreviations: BAI, Beck anxiety inventory; IQR, interquartile range; s.d., standard deviation.

${ }^{a}$ Based on $\chi^{2}$ test for dichotomous and categorical variables and independent samples $t$-test for continuous variables testing the difference between men and women; because of non-normal distributions, the Mann-Whitney $U$-test was used to test differences in raw inflammatory levels.

cardiovascular disease were $1.62(\mathrm{~s} . \mathrm{e}=1.11)$, illustrating the clinical relevance of this finding. Excluding persons reporting having had a cold or fever in the week before blood draw $(N=513)$, yielded similar findings (age of onset after 50 years versus controls: 1.73 (s.e. $=1.20$ ) versus 1.18 (s.e. $=1.05$ ) $\mathrm{mgl}^{-1}, P=0.04$, Cohen's $d=0.35$ ). Results were also similar when the analysis of Figure 1 was restricted to the sample of persons aged 50 years or above $(N=589$; age of onset after 50 years versus controls: 2.05 (s.e. $=1.16$ ) versus 1.35 (s.e. $=1.08$ ) $\mathrm{mg} \mathrm{I}^{-1}, P=0.01$, Cohen's $d=0.40$ ), underlining that higher CRP in those with an age of onset of 50 years or above was not due to the higher age itself in these persons. Last, in a post-hoc analysis, we directly compared CRP levels between persons with a late versus early onset of anxiety disorder at a cutoff of 50 years, and found significantly higher CRP levels in the late onset group (1.91 (s.e. $=1.19)$ versus 1.35 (s.e. $=1.03$ ) $\mathrm{mgl}^{-1}, P=0.05$, Cohen's $d=0.30$ ).

\section{Discussion}

The current study is one of the first and the largest to date to examine the association between anxiety disorders and inflammation. The results show that men with a current anxiety disorder have somewhat increased levels of CRP, even after taking a large set of lifestyle and disease factors into account. Elevated levels of CRP were in particular found in those persons with a late onset of the anxiety disorder.

Our results are in line with the few previous studies examining the relationship between anxiety symptoms or disorders with inflammation. Available evidence until now was limited to assessing anxiety symptoms in the general population, ${ }^{14,15}$ confined to specific anxiety disorders in small clinical samples ${ }^{16-18}$ or in a heart disease population. ${ }^{19}$ Our study adds to the literature by showing that elevated CRP levels can be found among several common anxiety disorders in a relatively large cohort of anxious persons and controls, specifically in those with a later onset of the anxiety disorder. CRP levels were in particular elevated among men with anxiety disorders, which is in line with the large-scale study by Liukkonen et al., ${ }^{15}$ which showed an association between anxiety symptoms and CRP only in men. In contrast, Pitsavos et al. ${ }^{14}$ found associations between an anxiety symptoms score and CRP levels in both men and women. Persons included in the study by Pitsavos et al. were much older 
Table 2 Adjusted mean inflammatory marker levels across anxiety groups

\begin{tabular}{|c|c|c|c|c|c|c|c|c|c|}
\hline & \multicolumn{3}{|c|}{ Controls } & \multicolumn{3}{|c|}{ Remitted anxiety disorder } & \multicolumn{3}{|c|}{ Current anxiety disorder } \\
\hline & Mean & (s.e.) & $\mathrm{P}$ & Mean & (s.e.) & $\mathrm{P}$ & Mean & (s.e.) & $\mathrm{P}$ \\
\hline \multicolumn{10}{|c|}{$C R P, \mathrm{mgl}^{-1 \mathrm{a}}$} \\
\hline $\begin{array}{l}\text { Total sample } \\
\text { Unadjusted }^{\mathrm{b}} \\
\text { Adjusted }^{\mathrm{c}} \\
\text { Adjusted }^{\mathrm{d}} \\
\text { Sex-interaction }^{\mathrm{e}}\end{array}$ & $\begin{array}{l}N=556 \\
1.11 \\
1.20 \\
1.28\end{array}$ & $\begin{array}{l}(1.05) \\
(1.05) \\
(1.05)\end{array}$ & $\begin{array}{l}\text { Ref } \\
\text { Ref } \\
\text { Ref }\end{array}$ & $\begin{array}{l}1.25 \\
1.24 \\
1.21\end{array}$ & $\begin{array}{c}N=459 \\
(1.06) \\
(1.06) \\
(1.05)\end{array}$ & $\begin{array}{l}0.14 \\
0.66 \\
0.46 \\
0.57\end{array}$ & $\begin{array}{l}1.36 \\
1.33 \\
1.30\end{array}$ & $\begin{array}{l}N=1273 \\
(1.04) \\
(1.03) \\
(1.03)\end{array}$ & $\begin{array}{l}0.001 \\
0.10 \\
0.74 \\
0.002\end{array}$ \\
\hline $\begin{array}{l}\text { Men } \\
\text { Unadjusted }^{\mathrm{b}} \\
\text { Adjusted }^{\mathrm{c}} \\
\text { Adjusted }^{\mathrm{d}}\end{array}$ & $\begin{array}{l}N=219 \\
0.84 \\
0.90 \\
0.98\end{array}$ & $\begin{array}{l}(1.08) \\
(1.08) \\
(1.07)\end{array}$ & $\begin{array}{l}\text { Ref } \\
\text { Ref } \\
\text { Ref }\end{array}$ & $\begin{array}{l}1.02 \\
0.98 \\
0.96\end{array}$ & $\begin{array}{l}N=131 \\
(1.11) \\
(1.10) \\
(1.09)\end{array}$ & $\begin{array}{l}0.15 \\
0.50 \\
0.82\end{array}$ & $\begin{array}{l}1.25 \\
1.22 \\
1.18\end{array}$ & $\begin{array}{l}N=407 \\
(1.06) \\
(1.06) \\
(1.05)\end{array}$ & $\begin{array}{c}<0.001 \\
0.002 \\
0.04\end{array}$ \\
\hline $\begin{array}{l}\text { Women } \\
\text { Unadjusted }^{\mathrm{b}} \\
\text { Adjusted }^{\mathrm{c}} \\
\text { Adjusted }^{\mathrm{d}}\end{array}$ & $\begin{array}{l}N=337 \\
1.34 \\
1.41 \\
1.48\end{array}$ & $\begin{array}{l}(1.07) \\
(1.07) \\
(1.06)\end{array}$ & $\begin{array}{l}\text { Ref } \\
\text { Ref } \\
\text { Ref }\end{array}$ & $\begin{array}{c}1.35 \\
1.40 \\
1.36 \\
I L-6, p g\end{array}$ & $\begin{array}{c}N=328 \\
(1.07) \\
(1.07) \\
(1.06)\end{array}$ & $\begin{array}{l}0.89 \\
0.95 \\
0.35\end{array}$ & $\begin{array}{l}1.42 \\
1.37 \\
1.36\end{array}$ & $\begin{array}{l}N=866 \\
(1.04) \\
(1.04) \\
(1.04)\end{array}$ & $\begin{array}{l}0.46 \\
0.74 \\
0.26\end{array}$ \\
\hline $\begin{array}{l}\text { Total sample } \\
\text { Unadjusted }^{\mathrm{b}} \\
\text { Adjusted }^{\mathrm{c}} \\
\text { Adjusted }^{\mathrm{d}} \\
\text { Sex-interaction }^{\mathrm{e}}\end{array}$ & $\begin{array}{l}N=556 \\
0.73 \\
0.75 \\
0.79\end{array}$ & $\begin{array}{l}(1.04) \\
(1.04) \\
(1.04)\end{array}$ & $\begin{array}{l}\text { Ref } \\
\text { Ref } \\
\text { Ref }\end{array}$ & $\begin{array}{l}0.71 \\
0.70 \\
0.70\end{array}$ & $\begin{array}{c}N=459 \\
(1.05) \\
(1.05) \\
(1.04)\end{array}$ & $\begin{array}{l}0.78 \\
0.30 \\
0.06 \\
0.47\end{array}$ & $\begin{array}{l}0.79 \\
0.78 \\
0.77\end{array}$ & $\begin{array}{l}N=1273 \\
(1.03) \\
(1.03) \\
(1.03)\end{array}$ & $\begin{array}{l}0.08 \\
0.35 \\
0.63 \\
0.40\end{array}$ \\
\hline \multicolumn{10}{|c|}{$T N F-\alpha, p g m l^{-1 a}$} \\
\hline $\begin{array}{l}\text { Unadjusted }^{\mathrm{b}} \\
\text { Adjusted }^{\mathrm{c}} \\
\text { Adjusted }^{\mathrm{d}} \\
\text { Sex-interaction }^{\mathrm{e}}\end{array}$ & $\begin{array}{l}0.82 \\
0.84 \\
0.85\end{array}$ & $\begin{array}{l}(1.03) \\
(1.03) \\
(1.03)\end{array}$ & $\begin{array}{l}\text { Ref } \\
\text { Ref } \\
\text { Ref }\end{array}$ & $\begin{array}{l}0.81 \\
0.81 \\
0.82\end{array}$ & $\begin{array}{l}(1.03) \\
(1.03) \\
(1.03)\end{array}$ & $\begin{array}{l}0.75 \\
0.43 \\
0.35 \\
0.92\end{array}$ & $\begin{array}{l}0.83 \\
0.83 \\
0.82\end{array}$ & $\begin{array}{l}(1.02) \\
(1.02) \\
(1.02)\end{array}$ & $\begin{array}{l}0.73 \\
0.75 \\
0.41 \\
0.87\end{array}$ \\
\hline
\end{tabular}

Abbreviations: CRP, C-reactive protein; IL-6, interleukin-6; TNF- $\alpha$, tumor necrosis factor- $\alpha$.

${ }^{a}$ To normalize distributions CRP, IL- 6 and TNF- $\alpha$ were In-transformed; for interpretation purposes presented means are back-transformed. ${ }^{b}$ Based on analyses of variance. ${ }^{\circ}$ Based on analyses of covariance adjusted for (sex,) age and education. ${ }^{\mathrm{d} B a s e d}$ on analyses of covariance additionally adjusted for smoking status, alcohol intake, physical activity, body mass index, cardiovascular disease, diabetes, number of other chronic diseases, statins and anti-inflammatory medication. e Sex-interactions are based on the fully adjusted model including anxiety status and a sex $\times$ anxiety status interaction term.

Table 3 Association ${ }^{a}$ of anxiety characteristics with inflammatory marker levels in persons with current anxiety disorders $(N=1273)$

\begin{tabular}{|c|c|c|c|c|c|c|c|}
\hline \multirow[t]{2}{*}{ Anxiety characteristic } & \multirow[t]{2}{*}{$\mathrm{N}^{\mathrm{c}}$} & \multicolumn{2}{|c|}{$\mathrm{CRP}^{\mathrm{b}}$} & \multicolumn{2}{|c|}{ IL- $6^{b}$} & \multicolumn{2}{|c|}{ TNF- $\alpha^{\mathrm{b}}$} \\
\hline & & $\beta$ & $\mathrm{P}$ & $\beta$ & $\mathrm{P}$ & $\beta$ & $\mathrm{P}$ \\
\hline Severity of anxiety symptoms (BAI) & 1254 & -0.027 & 0.31 & -0.017 & 0.54 & 0.017 & 0.57 \\
\hline Duration of anxiety symptoms & 1208 & -0.026 & 0.34 & -0.001 & 0.99 & 0.004 & 0.88 \\
\hline Age of anxiety disorder onset & 1254 & 0.053 & 0.05 & 0.019 & 0.53 & 0.044 & 0.15 \\
\hline Generalized anxiety disorder & 1273 & 0.021 & 0.40 & 0.036 & 0.19 & 0.042 & 0.13 \\
\hline Social phobia & 1273 & -0.053 & 0.04 & -0.052 & 0.05 & 0.013 & 0.64 \\
\hline Panic disorder and/or agoraphobia & 1273 & 0.011 & 0.66 & 0.026 & 0.35 & -0.014 & 0.62 \\
\hline Co-morbid depressive disorder & 1273 & -0.016 & 0.53 & -0.026 & 0.34 & 0.025 & 0.37 \\
\hline
\end{tabular}

Abbreviations: BAI, Beck anxiety inventory; CRP, C-reactive protein; IL-6, interleukin-6; TNF- $\alpha$, tumor necrosis factor- $\alpha$.

a Based on linear regression analyses adjusted for sex, age, education, smoking status, alcohol intake, physical activity, body mass index, cardiovascular disease, diabetes, number of other chronic diseases, statins and anti-inflammatory medication. ${ }^{\mathrm{b}} \mathrm{CRP}, \mathrm{IL}-6$ and TNF- $\alpha$ were In-transformed to normalize distributions. ${ }^{\mathrm{C}} \mathrm{N}$ differs due to missing values on some of the anxiety characteristics.

(18-89 years; mean age 45 years) than those in the study by Liukkonen et al. (all 31 years old), and slightly older than those in the present study (18-65 years; mean age 42 years). Perhaps sex differences become less clear with increasing age, as a result of hormonal changes across the lifespan of women, which affect inflammation levels. ${ }^{36}$ This could be in line with our finding that CRP levels were elevated in both men and women with a late onset of anxiety disorders.

Our findings with respect to anxiety disorders are also very comparable to our earlier findings regarding depressive disorders and inflammation. ${ }^{33}$ In that study, we found elevated inflammation, specifically CRP, in depressed men, especially among those with a later depression onset. The effect sizes for CRP in men with a current disorder are also comparable for anxiety (Cohen's $d=0.18$ ) and depressive (Cohen's $d=0.21$ ) disorders. A trend for association with IL-6, which was found for current depressive disorders in men, was not found for current anxiety disorders. Of note is that in persons with an anxiety disorder, a co-morbid depressive disorder was not associated with higher inflammation levels, suggesting that the effects found for anxiety disorders are independent of depression.

In line with our previous findings for current depressive disorders, ${ }^{33}$ CRP levels were in particular elevated among 


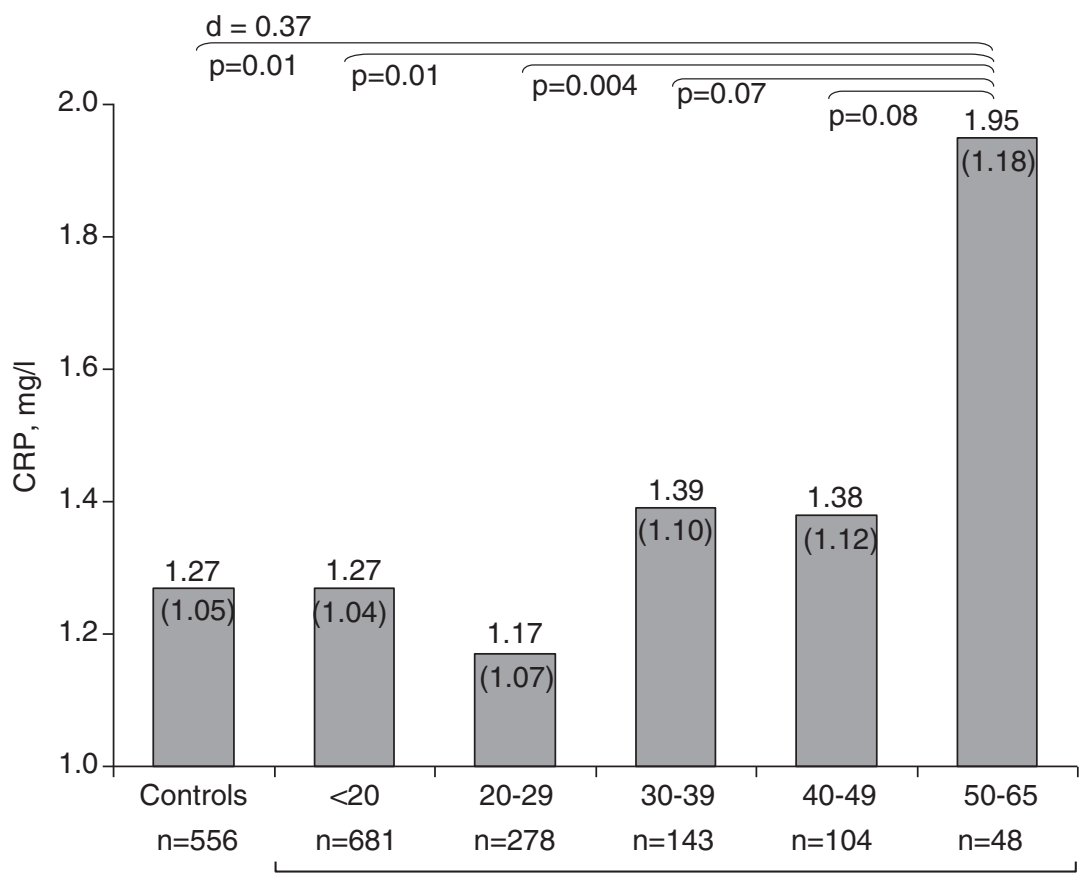

Age of anxiety disorder onset

Figure 1 Adjusted mean (s.e.) C-reactive protein (CRP) levels across controls and age of anxiety disorder onset groups. Based on analysis of covariance adjusted for sex, age, education, smoking status, alcohol intake, physical activity, body mass index, cardiovascular disease, diabetes, number of other chronic diseases, statins and antiinflammatory medication; to normalize distributions, CRP was In-transformed and for interpretation purposes presented means were back-transformed.

persons with a later onset of anxiety disorders. In contrast, characteristics that are more often associated with an early age of onset, such as higher severity and longer duration were not associated with increased inflammation. Also, in our sample, women had an earlier age of anxiety disorder onset than men, possibly contributing to the lack of an overall association between anxiety disorders and inflammation in women. Furthermore, we found that CRP levels were lowest among persons with social phobia when compared with other anxiety disorders, in particular in women. Social phobia has been reported to have a much earlier age of onset compared with generalized anxiety disorder or panic disorder, ${ }^{37}$ which was confirmed in our sample (16.6 versus 25.9 years, $P<0.001)$. To our knowledge, no other study has yet examined the association between social phobia and inflammation. In our study, only nine persons with social phobia had an disorder onset at or after 50 years. Therefore, low inflammation levels in persons with social phobia cannot explain our findings for elevated CRP levels in persons with an age of anxiety disorder onset after 50 years. A recent study by Copeland et al. ${ }^{38}$ showed that, after taking health-related behaviors into account, generalized anxiety disorder was not associated with elevated CRP levels among children and adolescents. These findings argue against the idea that the inflammation-anxiety association is merely a result of acute stress experienced in anxiety disorders. Although we cannot make inferences about etiology based on our cross-sectional analyses, our current findings are in line with the growing evidence suggesting a distinct etiology involving vascular/ metabolic/inflammatory factors in depression or anxiety disorders with an onset later in life. ${ }^{39-42}$ Possibly, accumulating psychological and physical stress across the life-span might induce immunological changes ${ }^{24}$ that eventually results in depression and anxiety.

In our previous report, ${ }^{33}$ we had found differences in inflammation levels among different classes of antidepressant medication use, which was confirmed for higher CRP in TCA/ TeCA users within our present sample of persons with current anxiety disorders. Excluding persons using TCA or TeCA, resulted in a slightly weaker effect size for the association between current anxiety disorder and CRP in men. This might suggest that the elevated CRP levels in men with current anxiety disorders are for some part due to use of TCA/TeCA. On the other hand, persons using TCA/TeCA might represent the more severe cases of anxiety disorders, in which case exclusion of these persons leads to an underestimation of the association. Adjustment for TCA/TeCA use had no effect on our findings for age of anxiety disorder onset, suggesting that late-onset anxiety disorders are independently associated with higher levels of CRP.

What are the clinical implications of our findings? First, our finding of increased CRP levels in particularly those with a late onset of the anxiety disorder might implicate the existence of a specific late-onset anxiety subtype with a distinct etiology. As we have found similar results for depression ${ }^{33}$ and because depression and anxiety are highly co-morbid disorders, ${ }^{11}$ this might suggest that depression and anxiety with a late onset share a similar etiology and represent one particular group of disorders, which might be more distinct from other depressive or anxiety disorders, which present earlier in life. As we can only speculate on etiology based on our cross-sectional research, longitudinal research is needed to validate the 
existence of an etiologically distinct late-onset subtype. Second, if confirmed, a distinct etiology for late-onset disorders implicates different treatment strategies for this subgroup. Perhaps anti-inflammatory medication or lifestyle interventions, such as exercise, for which (some) evidence exists that they normalize immune and metabolic dysregulation, ${ }^{43}$ as well as improve depressive symptoms to some degree, ${ }^{44,45}$ could be beneficial in persons with late onset anxiety disorders as well.

Our study has some important strengths such as a large sample size, assessment of multiple inflammatory markers, clinical diagnoses of several anxiety disorders, adequate adjustment for potential confounders and the ability to examine the role of anxiety characteristics. However, some limitations need to be acknowledged. As our data are crosssectional, we cannot make any inferences about the direction of the association. Also, although we adjusted for a large set of possible confounding factors, unmeasured poor lifestyle behaviors or health factors may be the explaining link between inflammation and anxiety disorders. For instance, subclinical cardiovascular disease could possibly precede both inflammation and anxiety. On the other hand, subclinical disease may be one pathway of how inflammation leads to anxiety in later life. Longitudinal studies are needed to investigate whether immune dysregulation is a precursor or the result of anxiety, or whether this relationship is bidirectional. Further, like most other studies, we assessed circulating levels of inflammatory markers, which show a high degree of intraindividual variation that could explain the rather modest overall associations between anxiety disorders and inflammation in our study.

In conclusion, our results show that low-grade systemic inflammation is present in men with anxiety disorders. Elevated inflammation is in particular found in both men and women with the onset of anxiety disorder later in life. Longitudinal studies are needed to confirm inflammation as an etiological factor in anxiety disorders with a late-life onset, followed by intervention trials investigating new treatment strategies (for example, antiinflammatory medication, lifestyle interventions) for this subset of persons with late-onset anxiety.

\section{Conflict of interest}

The authors declare no conflict of interest.

Acknowledgements. The infrastructure for the NESDA study (http:/ www.nesda.nl) is funded through the Geestkracht program of the Netherlands Organisation for Health Research and Development (Zon-Mw, grant number 10000-1002) and is supported by participating universities and mental health care organizations (VU University Medical Center, GGZ inGeest, Arkin, Leiden University Medical Center, GGZ Rivierduinen, University Medical Center Groningen, Lentis, GGZ Friesland, GGZ Drenthe, Institute for Quality of Health Care (IQ Healthcare), the Netherlands Institute for Health Services Research (NIVEL) and the Netherlands Institute of Mental Health and Addiction (Trimbos)). NV was supported through a fellowship from the EMGO Institute for Health and Care Research and BP through a VICl grant (NWO grant g1811602). Assaying of inflammatory markers was supported by the Neuroscience Campus Amsterdam.

1. Kessler RC, Chiu WT, Demler O, Merikangas KR, Walters EE. Prevalence, severity, and comorbidity of 12-month DSM-IV disorders in the National Comorbidity Survey Replication. Arch Gen Psychiatry 2005; 62: 617-627.
2. Buist-Bouwman MA, de GR, Vollebergh WA, Alonso J, Bruffaerts R, Ormel J. Functional disability of mental disorders and comparison with physical disorders: a study among the general population of six European countries. Acta Psychiatr Scand 2006; 113: 492-500.

3. Seldenrijk A, Vogelzangs N, van Hout HP, van Marwijk HW, Diamant M, Penninx BW. Depressive and anxiety disorders and risk of subclinical atherosclerosis Findings from the Netherlands Study of Depression and Anxiety (NESDA). J Psychosom Res 2010; 69: 203-210.

4. Carroll D, Phillips AC, Thomas GN, Gale CR, Deary I, Batty GD. Generalized anxiety disorder is associated with metabolic syndrome in the Vietnam experience study. Biol Psychiatry 2009; 66: 91-93.

5. Roest AM, Martens EJ, de JP, Denollet J. Anxiety and risk of incident coronary heart disease: a meta-analysis. J Am Coll Cardiol 2010; 56: 38-46.

6. Vogelzangs N, Seldenrijk A, Beekman AT, van Hout HP, de JP, Penninx BW. Cardiovascular disease in persons with depressive and anxiety disorders. J Affect Disord 2010; 125: 241-248.

7. Libby P. Inflammation in atherosclerosis. Nature 2002; 420: 868-874.

8. Sutherland JP, McKinley B, Eckel RH. The metabolic syndrome and inflammation. Metab Syndr Relat Disord 2004; 2: 82-104.

9. Willerson JT, Ridker PM. Inflammation as a cardiovascular risk factor. Circulation 2004 109(21 Suppl 1): II2-10.

10. Huffman JC, Celano CM, Januzzi JL. The relationship between depression, anxiety, and cardiovascular outcomes in patients with acute coronary syndromes. Neuropsychiatr Dis Treat 2010; 6: 123-136.

11. Lamers $F$, van OP, Comijs HC, Smit JH, Spinhoven $P$, van Balkom AJ et al. Comorbidity patterns of anxiety and depressive disorders in a large cohort study: the Netherlands Study of Depression and Anxiety (NESDA). J Clin Psychiatry 2011; 72 : 341-348

12. Howren MB, Lamkin DM, Suls J. Associations of depression with C-reactive protein, IL-1, and IL-6: a meta-analysis. Psychosom Med 2009; 71: 171-186.

13. Dowlati Y, Herrmann N, Swardfager W, Liu H, Sham L, Reim EK et al. A meta-analysis of cytokines in major depression. Biol Psychiatry 2010; 67: 446-457.

14. Pitsavos C, Panagiotakos DB, Papageorgiou C, Tsetsekou E, Soldatos C, Stefanadis C. Anxiety in relation to inflammation and coagulation markers, among healthy adults: the ATTICA study. Atherosclerosis 2006; 185: 320-326.

15. Liukkonen T, Rasanen P, Jokelainen J, Leinonen M, Jarvelin MR, Meyer-Rochow VB et al. The association between anxiety and C-reactive protein (CRP) levels: results from the Northern Finland 1966 birth cohort study. Eur Psychiatry 2011; 26: 363-369.

16. Gill JM, Saligan L, Woods S, Page G. PTSD is associated with an excess of inflammatory immune activities. Perspect Psychiatr Care 2009; 45: 262-277.

17. Spitzer C, Barnow S, Volzke H, Wallaschofski H, John U, Freyberger HJ et al. Association of posttraumatic stress disorder with low-grade elevation of C-reactive protein: evidence from the general population. J Psychiatr Res 2010; 44: 15-21.

18. Hoge EA, Brandstetter K, Moshier S, Pollack MH, Wong KK, Simon NM. Broad spectrum of cytokine abnormalities in panic disorder and posttraumatic stress disorder. Depress Anxiety 2009; 26: 447-455

19. Bankier B, Barajas J, Martinez-Rumayor A, Januzzi JL. Association between C-reactive protein and generalized anxiety disorder in stable coronary heart disease patients. Eur Heart J 2008; 29: 2212-2217.

20. Hamer M, Gibson EL, Vuononvirta R, Williams E, Steptoe A. Inflammatory and hemostatic responses to repeated mental stress: individual stability and habituation over time. Brain Behav Immun 2006; 20: 456-459.

21. Leonard BE, Myint A. The psychoneuroimmunology of depression. Hum Psychopharmacol 2009; 24: 165-175.

22. Miller GE, Cohen S, Ritchey AK. Chronic psychological stress and the regulation of pro-inflammatory cytokines: a glucocorticoid-resistance model. Health Psychol 2002; 21: 531-541.

23. Wirtz PH, von KR, Schnorpfeil P, Ehlert U, Frey K, Fischer JE. Reduced glucocorticoid sensitivity of monocyte interleukin- 6 production in male industrial employees who are vitally exhausted. Psychosom Med 2003; 65: 672-678.

24. Bauer ME. Chronic stress and immunosenescence: a review. Neuroimmunomodulation 2008; 15: 241-250.

25. Penninx BW, Beekman AT, Smit JH, Zitman FG, Nolen WA, Spinhoven $P$ et al. The Netherlands Study of Depression and Anxiety (NESDA): rationale, objectives and methods. Int J Methods Psychiatr Res 2008; 17: 121-140.

26. American Psychiatric Association Diagnostic and Statistical Manual of Mental Disorders. 4th ed American Psychiatric Association: Washington, DC, 2001

27. Wittchen HU. Reliability and validity studies of the WHO-Composite International Diagnostic Interview (CIDI): a critical review. J Psychiatr Res 1994; 28: 57-84.

28. Beck AT, Epstein N, Brown G, Steer RA. An inventory for measuring clinical anxiety: psychometric properties. J Consult Clin Psychol 1988; 56: 893-897.

29. Kabacoff RI, Segal DL, Hersen M, Van Hasselt VB. Psychometric properties and diagnostic utility of the Beck Anxiety Inventory and the State-Trait Anxiety Inventory with older adult psychiatric outpatients. J Anxiety Disord 1997; 11: 33-47.

30. Lyketsos CG, Nestadt G, Cwi J, Heithoff K, Eaton WW. The life chart interview: a standardized method to describe the course of psychopathology. Int J Methods Psychiatric Res 1994; 4: 143-155. 
31. Eaton WW, Shao H, Nestadt G, Lee HB, Bienvenu OJ, Zandi P. Population-based study of first onset and chronicity in major depressive disorder. Arch Gen Psychiatry 2008; 65: 513-520.

32. Martyn KK, Belli RF. Retrospective data collection using event history calendars. Nurs Res 2002; 51: 270-274.

33. Vogelzangs N, Duivis HE, Beekman AT, Kluft C, Neuteboom J, Hoogendijk W et al. Association of depressive disorders, depression characteristics and antidepressant medication with inflammation. Transl Psychiatry 2012; 2: e79.

34. Craig CL, Marshall AL, Sjostrom M, Bauman AE, Booth ML, Ainsworth BE et al. International physical activity questionnaire: 12-country reliability and validity. Med Sci Sports Exerc 2003; 35: 1381-1395.

35. WHO. Collaborating Centre for Drug Statistics Methodology. Anatomical Therapeutic Chemical Classification. World Health Organization: Geneva, 2007.

36. Cushman M, Legault C, Barrett-Connor E, Stefanick ML, Kessler C, Judd HL et al. Effect of postmenopausal hormones on inflammation-sensitive proteins: the Postmenopausal Estrogen/Progestin Interventions (PEPI) Study. Circulation 1999; 100: 717-722.

37. Kessler RC, Berglund P, Demler O, Jin R, Merikangas KR, Walters EE. Lifetime prevalence and age-of-onset distributions of DSM-IV disorders in the National Comorbidity Survey Replication. Arch Gen Psychiatry 2005; 62: 593-602.

38. Copeland WE, Shanahan L, Worthman C, Angold A, Costello EJ. Generalized anxiety and C-reactive protein levels: a prospective, longitudinal analysis. Psychol Med 2012; 42: 2641-2650.

39. Alexopoulos GS, Meyers BS, Young RC, Campbell S, Silbersweig D, Charlson M. 'Vascular depression' hypothesis. Arch Gen Psychiatry 1997; 54: 915-922.
40. Kendler KS, Fiske A, Gardner CO, Gatz M. Delineation of two genetic pathways to major depression. Biol Psychiatry 2009; 65: 808-811.

41. Seldenrijk A, van Hout HP, van Marwijk HW, de GE, Gort J, Rustemeijer $C$ et al. Carotid atherosclerosis in depression and anxiety: Associations for age of depression onset. World J Biol Psychiatry 2011; 12: 549-558.

42. Smith PJ, Blumenthal JA, Babyak MA, Doraiswamy PM, Hinderliter A, Hoffman BM et al. Intima-media thickness and age of first depressive episode. Biol Psychol 2009; 80 361-364.

43. You T, Nicklas BJ. Effects of exercise on adipokines and the metabolic syndrome. Curr Diab Rep 2008; 8: 7-11.

44. Mead GE, Morley W, Campbell P, Greig CA, McMurdo M, Lawlor DA. Exercise for depression. Cochrane Database Syst Rev 2009; (3):CD004366.

45. Muller N, Schwarz MJ, Dehning S, Douhe A, Cerovecki A, Goldstein-Muller B et al. The cyclooxygenase-2 inhibitor celecoxib has therapeutic effects in major depression: results of a double-blind, randomized, placebo controlled, add-on pilot study to reboxetine. Mol Psychiatry 2006; 11: 680-684

(c) (i) (8) $\odot$ Translational Psychiatry is an open-access journal published by Nature Publishing Group. This work is licensed under a Creative Commons Attribution-NonCommercialNoDerivs 3.0 Unported License. To view a copy of this license, visit http://creativecommons.org/licenses/by-nc-nd/3.0/ 